\title{
The Immunopathology of Intraocular Tumour Rejection
}

\author{
J. Y. NIEDERKORN \\ Dallas, Texas, USA
}

\begin{abstract}
Summary
The immunopathology of intraocular tumour rejection was studied using two transplantable tumours in mice. UV5C25 fibrosarcoma and P91 mastocytoma express tumour specific antigens that elicit strong systemic $T$ cell-mediated immune responses following intracameral transplantation. Although both tumours underwent immunological rejection, the immunopathological sequelae of tumour rejection differed markedly. P91 tumour rejection was accompanied by bulk ischaemic necrosis, microvascular damage, and extensive damage to innocent bystander cells. A large body of experimental evidence indicated that this ischaemic necrotic pattern of intraocular tumour rejection was the result of a delayed-type hypersensitivity (DTH) effector mechanism. By contrast, intraocular UV5C25 tumour rejection occurred by piecemeal necrosis and did not culminate in injury to juxtaposed normal host tissues. The results of in vitro and in vivo experiments strongly indicated a role for cytotoxic T lymphocyte (CTL) mediated mechanisms in the piecemeal necrotic pattern of tumour rejection. Collectively, the results indicated that although tumour bearing hosts possessed both CTL and DTH effector mechanisms, only one form of T cell-mediated immunity prevailed within the intraocular milieu.
\end{abstract}

The eye has been likened to an immunological microcosm in which virtually all forms of immunological events can take place, and occasionally do so with disastrous consequences. ${ }^{1}$ Indeed, inflammatory diseases are important causes of blindness. The possibility that inflammatory eye diseases, such as uveitis, were immune-mediated was proposed over 80 years ago by Elschnig. ${ }^{2}$ Subsequently, literally scores of studies have produced compelling evidence that at least some forms of idiopathic uveitis are immune-mediated.

Recent advances in the field of tumour immunology offer glimmers of optimism for the eventual implementation of immunotherapy for a variety of neoplasms, especially malignant melanoma. Impressive results with the use of tumour infiltrating lymphocytes (TIL) in the treatment of metastatic cutaneous melanoma and the recent NIH approval to implement gene therapy into current protocols for TIL immunotherapy are a testament to the rapid progress in this field. ${ }^{3}$ The swift development and application of novel immunotherapeutic procedures underscore the urgency for understanding the immunobiology of intraocular tumours. Unlike other anatomical regions, the intraocular milieu possesses unique biological and immunological characteristics that can present important complications for implementing immunotherapeutic modalities. Our ongoing studies, 
summarised here, have attempted to characterise the immunobiology of intraocular tumour rejection.

It is clear that protective immune responses that shield the eye from infectious diseases can unwittingly produce deleterious immunemediated damage to innocent bystander cells within the eye. Historically, the basic immune mechanisms of tissue damage have been categorised into four basic types:

(i) type I = anaphylactic;

(ii) type II = cytotoxic;

(iii) type III = immune complexes; and

(iv) cell-mediated.

The first three categories of immunemediated damage involve antibody. Type I hypersensitivity involves the degranulation of mast cells by antigen specific IgE antibodies. Classical allergic or immediate hypersensitivity responses are loosely categorised as a form of immune mediated tissue damage. Type II hypersensitivity reactions produce direct tissue damage by cytolytic antibodies and the fixation of complement. Type III hypersensitivity injury occurs indirectly. Antigen-antibody complexes activate the complement cascade which in turn culminates in a constellation of events including the recruitment of activated neutrophils, deposition of fibrin, release of leukocytic lysosomal enzymes, and increased vascular permeability. Type IV hypersensitivity produces tissue destruction by one of two independent $\mathrm{T}$ cell-mediated processes. Cytotoxic T lymphocytes (CTL) can produce damage by direct cytolysis of the target cell. This form of immune reactivity is crucial in the elimination of infectious virus. Virally infected cells are recognised by virusspecific CTL that directly lyse the infected host cell via recognition of the viral antigens displayed on the cell membrane of the infected host cell. Cytolysis requires the recognition of both the relevant viral antigen and the presence of the host's unique class I major histocompatibility complex (MHC) antigens concomitantly displayed in association with the viral antigens. The MHC restriction of CTL-mediated cytolysis of virally infected cells ensures the cytolysis is limited to infected cells of host origin. As a result, juxtaposed, noninfected host cells are spared and cytolysis is narrowly focused to affect only the infected cell population. Direct cell-mediated cytotoxicity (CMC) can also produce piecemeal killing of tumour cells. CTL and natural killer (NK) cells can recognise neoplastic cells, bind directly to such target cells, and release lytic granules directly onto the target cell membrane producing almost immediate death of the bound cell. Direct cell-mediated cytotoxicity produces prompt killing of renegade cells without inflicting damage to innocent bystander cells. Cell-mediated hypersensitivity also includes delayed-type hypersensitivity (DTH) responses. Unlike cell-mediated cytotoxicity, DTH effector responses culminate in extensive injury to juxtaposed normal cells. Upon exposure to relevant antigen, primed $\mathrm{T}$ cells (usually of the $\mathrm{CD} 4+, \mathrm{Th} 1$ phenotype) elaborate a potpourri of cytokines which stimulate the migration, proliferation, and activation of second level effector cells including macrophages, neutrophils, lymphocytes, monocytes, and occasionally, eosinophils. The ensuing inflammatory response carries a heavy burden of damage to normal juxtaposed cells. Thus, unlike CMC responses, DTH reactions disrupt the integrity of the surrounding normal tissues.

\section{Immunological Rejection of Intraocular Tumours: Ischaemic Necrotic Pattern}

In order to study immunologic regulation within the eye, we have relied heavily on murine models which permit immunologic and immunogenetic analyses not possible in any other laboratory animal. During the course of these studies it has become apparent that two distinct categories of spontaneous tumour rejection are distinguishable. ${ }^{4-8}$

The first pattern culminates in ischaemic necrosis en masse and the complete atrophy of the affected eye. This model involves the use of a highly immunogenic mastocytoma (P91) which expresses tumour specific antigens that are readily recognised by the syngeneic DBA/2 mouse host. ${ }^{4}$ Following intracameral transplantation, the P91 tumour grew progressively for approximately 14 days. However, beginning at approximately day 14 , significant damage of vascular endothelial cells of the blood vessels feeding the tumour occurred. ${ }^{4,5,7}$ Vascular endothelial damage 
was followed by necrosis of the tumour en masse. Importantly, there was no evidence of piecemeal necrosis or direct cell-to-cell contact between host inflammatory cells and the tumour cells. Tumour resolution was complete by day 28 but resulted in extensive pathological sequelae to normal host tissues. Ischaemic necrosis resulted in scarring of the iris, cataract formation, and granulation scar tissue formation which in turn caused retinal detachment and ultimately, complete atrophy of the eye. Thus, the histopathological features of P91 tumour rejection were consistent with a DTH process based on:

(i) microvascular damage;

(ii) ischaemic bulk necrosis;

(iii) significant damage to innocent bystander cells;

(iv) the conspicuous absence of piecemeal necrosis of individual tumour cells.

The sudden onset and completion of tumour resolution could not be regarded as simply a case of the tumour outgrowing its blood supply since the same tumours grew progressively in $\mathrm{T}$ cell-deficient nude mice and sublethally irradiated (= leukopenic) euthymic DBA/2 mice (Figure 1). Thus, the ischaemic necrotic pattern of tumour rejection was $\mathrm{T}$ cell-dependent and radiosensitive.

The potential T cell-dependent, radiosensitive effector mechanisms that might produce tumour rejection include: CTL, DTH, or antibody. Additional experiments considered each of these effector modalities.

The possible role of antibody in ischaemic tumour rejection was evaluated in several experiments. If antibodies were involved, it should be possible to reproduce these sequelae by passively transferring hyperimmune serum to immunologically naive recipients. Accordingly, panels of sublethally irradiated $\mathrm{DBA} / 2$ mice and $\mathrm{T}$ cell-deficient nude BALB/c mice received multiple injections of hyperimmune serum from hosts who had rejected their intraocular P91 tumours. ${ }^{7}$ Even though the recipients received large quantities of hyperimmune serum, tumour growth was not arrested nor was there evidence of ischaemic necrosis of the affected eye (Table I).

Additional investigations considered the hypothesis that the complement cascade was involved in the ischaemic necrotic pattern of intraocular tumour rejection. This was examined by using the B10.D20Sn mouse strain which is incapable of producing the $\mathrm{C} 5 \mathrm{com}$ ponent of complement. ${ }^{9}$ Tumour rejection in this complement-deficient mouse strain was compared with that occurring in the B10.D2nSn counterpart which possesses normal complement activity. The results showed that tumour rejection was virtually identical in both mouse strains and culminated in ischaemic necrosis of the affected eye and tumour (Table II).

The aforementioned results indicated that antibody alone could not promote either tumour rejection or ischaemic necrosis of the tumour-containing eye. Likewise, the absence of complement did not affect the ischaemic necrotic pattern of tumour rejection. Additional experiments further supported the hypothesis that antibody was not involved in this form of immune-mediated ischaemic necrosis. These experiments were based on our previous observation that splenectomised mice fail to produce detectable antibody responses to antigens presented intracamerally. Accordingly, panels of splenectomised and normal DBA/2 mice received intracameral inoculations of P91 tumour cells. As expected, anti-tumour antibodies could be detected in only two of the 11 splenectomised hosts, yet ischaemic tumour rejection occurred in all hosts and resulted in phthisis of all of the eyes examined (Table II).

It is well known that tumour necrosis factor (TNF) can produce haemorrhagic necrosis of

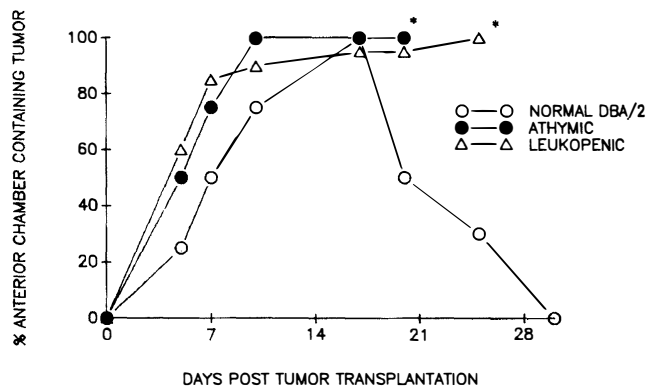

Fig. 1. 'Ischaemic necrotic' pattern of tumour rejection is $T$ cell-dependent and radiosensitive. Hosts were immunocompetent DBA/2 mice; T cell-deficient, athymic nude $B A L B / c$ mice; and leukopenic, sublethally (500 R) DBA/2 mice. Original experiments are described elsewhere. ${ }^{4}$ 
Table I. Tumour-specific antibody does not produce 'ischaemic necrotic' pattern of tumour rejection.

\begin{tabular}{lll}
\hline Host* & Treatment & Fate of Intraocular tumour \\
\hline T cell-deficient & Hyperimmune serum & Progressive growth \\
Leukopenic & Hyperimmune serum & Progressive growth \\
Normal & None & 'Ischaemic necrotic' rejection \\
\hline
\end{tabular}

*Details of experiment are described elsewhere. ${ }^{7} \mathrm{~T}$ cell-deficient mice $=$ athymic, nude $\mathrm{BALB} / \mathrm{c}$ mice; leukopenic mice $=$ sublethally $x$-irradiated $(500 \mathrm{rad}) \mathrm{DBA} / 2$ mice; normal $=\mathrm{DBA} / 2 \mathrm{mice}$.

mouse tumours. However, several experimental findings ruled out a role for TNF in the ischaemic necrosis of intraocular P91 tumours. In vitro assays revealed that P91 tumour cells were not susceptible to TNF elaborated by natural cytotoxic (NC) cells or by direct exposure to recombinant TNF. ${ }^{7}$

The results described above indicate that antibody was neither directly or indirectly involved in immune-mediated ischaemic necrosis of intraocular tumours. Recent studies in our laboratory have provided compelling evidence that CTL are not responsible for the observed ischaemic necrotic pattern of tumour rejection. Mice were treated systemically with anti-CD8 monoclonal antibody as a means of depleting the subpopulation of $\mathrm{T}$ lymphocytes that mediate classical cytotoxic T lymphocyte activity. ${ }^{10}$ The results showed that the antibody treatment completely abolished anti-tumour CTL activity (data not shown), yet the characteristic pattern and tempo of ischaemic necrotic tumour rejection was unaffected (Fig. 1).

The most plausible explanation to account for the ischaemic necrotic pattern of tumour rejection involves DTH-mediated processes. The weight of evidence indicates that rejection, although T-cell-dependent and radiosensitive, was not mediated by either antibody, TNF, or CTL. By the process of elimination, we are left to conclude that DTH is intimately involved in this process. The observed microvascular injury, ischaemic necrosis en masse, and the heavy burden of innocent bystander damage to normal ocular tissues are findings reminiscent of the lesions produced by severe DTH reactions in the skin ${ }^{11,12}$ and in rejecting tumours. ${ }^{13-15}$

Although the P91 tumour is not a model of human intraocular tumours, its unique pattern of rejection bears a curious similarity to the behaviour of retinoblastoma which is known occasionally to undergo spontaneous resolution. ${ }^{16,17}$ Some investigators have suggested that the spontaneous resolution of retinoblastoma is due to immunologic rejection. ${ }^{18}$ Moreover, reports of intraocular inflammation preceding the spontaneous resolution of retinoblastomas ${ }^{19}$ and the demonstration of tumour-specific antigens on several retinoblastoma cell lines ${ }^{20}$ suggest that the immune system may indeed recognise retinoblastomas and mediate their destruction. It bears noting that spontaneous resolution of retinoblastomas often culminates in phthisis bulbi, ${ }^{16}$ a condition not unlike the ischaemic necrotic pattern of intraocular P91 tumour rejection.

\section{Immunological Rejection of Intraocular Tumours: Piecemeal Necrotic Pattern}

In sharp contrast to the previously described pattern of tumour rejection is a second form of tumour resolution which culminates in the complete erradication of neoplasm without disturbing the anatomic integrity of the affected eye. The transplantable ultraviolet

Table II. 'Ischaemic necrotic' pattern of tumour rejection occurs in the absence antibody and complement. ${ }^{*}$

\begin{tabular}{llll}
\hline Host & Antibody & $\begin{array}{c}\text { Immune Status } \\
\text { Complement }\end{array}$ & Tumour Rejection \\
\hline DBA/2 & Positive & Deficient & 'Ischaemic' \\
B10.D2oSn & Positive & C5-deficient & 'Ischaemic' \\
B10.D2nSn & Positive & Normal & 'Ischaemic' \\
Splx.DBA/2 & Negative & Deficient & 'Ischaemic' \\
\hline
\end{tabular}

${ }^{*}$ Details of these experiments are described elsewhere. ${ }^{7}$ Splx.DBA/2 $=$ splenectomised DBA $/ 2$ mice. 


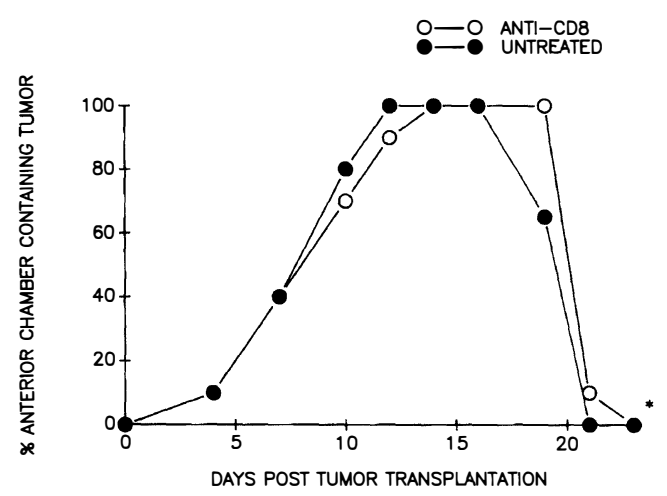

Fig. 2. Depletion of CTL activity does not alter tempo or immunopathological pattern of intraocular $P 91$ tumour rejection. $D B A / 2$ mice were depleted of $C D 8+$ $C T L$ by in vivo treatment with anti-CD8 antibody as described elsewhere. ${ }^{10}$ Tumour rejection occurred by an 'ischaemic necrotic' pattern that was clinically indistinguishable from previous experiments. ${ }^{4,7}$

radiation (UVR) induced fibrosarcoma, UV5C25 is a prototype of this form of intraocular tumour rejection. ${ }^{5,8}$ Intraocular UV5C25 tumours undergo a prolonged form of immunologic rejection in the eyes of syngeneic $\mathrm{BALB} / \mathrm{c}$ mice. ${ }^{5,8}$ Histopathological analysis of the resolving tumours revealed an impressive lymphocytic infiltrate accompanied by piecemeal necrosis of individual tumour cells. Direct binding of lymphocytes to individual tumour cells was a common feature of the resolving intraocular UV5C25 tumours and strongly suggested that tumour rejection was mediated by direct cytolysis by tumour-specific CTL. Immunological analyses were conducted to test this hypothesis.

Our approach to these studies was crudely patterned after 'Koch's Postulates' in which we sought to:

(i) isolate tumour-specific CTL from resolving tumours;

(ii) culture the isolated, tumour-specific CTL in vitro:

(iii) demonstrate their expression of surface markers characteristic of CTL;

(iv) demonstrate cytolytic antitumour activity in vitro;

(v) transfer the antitumour effect to naive recipients.

Each of these criteria has been confirmed and the results have been reported elsewhere. ${ }^{5,8}$ Fluorescein activated cell sorter (FACS) analysis of the tumour infiltrating lymphocyte (TIL) population indicated that the both CD4+ and CD8 $+\mathrm{T}$ cells were present in the resolving tumour mass (Fig. 3). However, in vitro functional assays indicated that the TIL displayed impressive direct cytotoxicity for UV5C25 tumours. Moreover, cytotoxicity was restricted to the relevant UV5C25 tumour cells and was not demonstrated against a battery of third-party tumour cell lines. ${ }^{8}$ Further experiments demonstrated that the isolated TIL could produce a similar piecemeal pattern of intraocular tumour rejection when adoptively transferred to immunologically impaired recipients. That is, TIL were mixed with viable tumour cells at a ratio of 1:10 and injected directly into the anterior chambers of immunosuppressed $\mathrm{BALB} / \mathrm{c}$ mice. For comparison, normal splenic lymphocytes from immunologically naive donors were similarly mixed with viable tumour cells and injected into similar hosts. As expected, the tumours grew vigorously and progressively in hosts given normal splenic lymphocytes. By contrast, tumours containing TIL underwent prompt rejection with minimal pathological sequelae. Importantly, the clinical characteristics of tumour rejection in the hosts treated with TIL were identical to those that occurred in normal immunocompetent hosts. ${ }^{8}$

If the hypothesis that CTL are responsible for the piecemeal necrotic pattern of tumour rejection is correct, then it should be possible

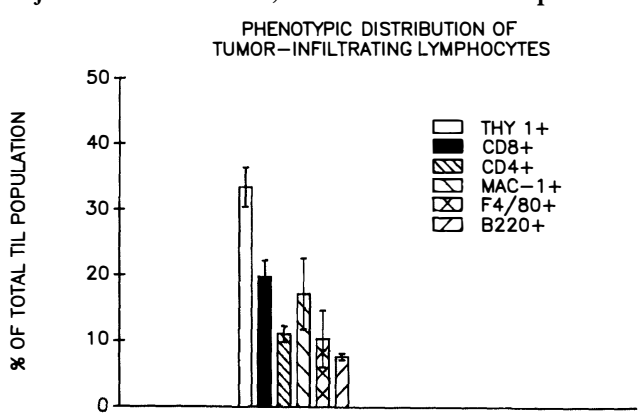

CELL SUBPOPULATIONS

Fig. 3. Phenotypic distribution of tumour infiltrating lymphocytes (TIL) isolated form intraocular tumours undergoing 'piecemeal necrotic' pattern of rejection. Details of this experiment are described elsewhere. ${ }^{8}$ Thy $1+=$ all $T$ cells; $C D 4+=$ helper/D TH T cell subset; CD8+ =CTL/suppressor $T$ cell subset; Mac-1 $=$ macrophages and granulocytes; F4/80 = macrophages; $B 220=B$ cells. 
to prevent rejection by eliminating CTL. This hypothesis was confirmed in recent experiments in which CTL populations were eliminated in vivo by treating BALB/c hosts with anti-CD8 antibody. In vivo treatment with anti-CD8 antibody resulted in a profound depletion of tumour specific CTL activity (data not shown) and the progressive growth of intraocular tumours (Fig. 4). Histological examination of the resolving intraocular tumours revealed an impressive absence of mononuclear inflammatory cells (data not shown). The eyes of anti-CD8 treated euthymic mice were histologically and grossly indistinguishable from $\mathrm{T}$ cell-deficient nude mice ${ }^{8}$ - in both categories of mice, mononuclear inflammatory cells were not detected at any time point examined. Thus, the weight of evidence favours the hypothesis that the piecemeal necrotic pattern of intraocular tumour rejection is mediated by CTL and that the DTH effector mechanism is excluded.

\section{Regulation of Immunologic Effector Mechanisms in the Eye}

The experimental findings summarised here underscore the complexity of intraocular immune regulation. The tumour models utilised for these studies were not intended to serve as counterparts to human uveal melanoma or retinoblastoma. Rather, they were employed as tools better to understand the immunobiology and immunopathology of intraocular tumours. Although both intraocular tumours induced potent tumour-speci-

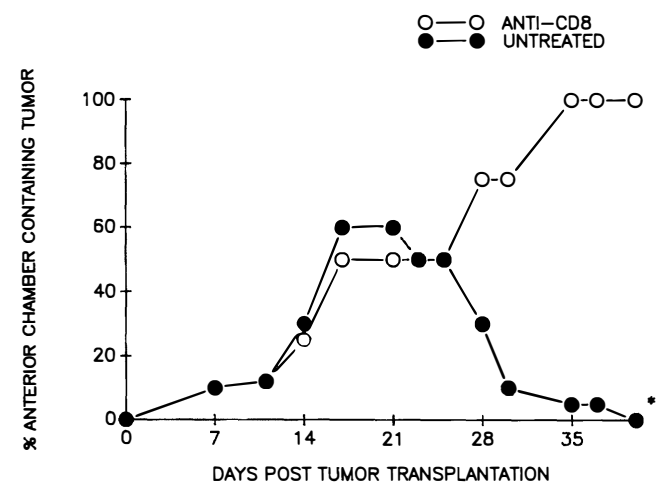

Fig. 4 Depletion of anti-tumour CTL prevents 'piecemeal necrotic' pattern of intraocular tumour rejection. Mice were depleted of CTL by in vivo treatment with anti-CD8 antibody. Clinical observations were made as described before..$^{5,8}$ fic CTL and DTH responses, only one immune mechanism was actively engaged within the intraocular milieu.

In the ischaemic necrotic pattern of tumour rejection, DTH effector mechanisms prevailed. The initiation of the DTH reaction in oculi was antigen specific; however, the effector arm was non-specific. The most likely scenario is that activated DTH effector T cell population elaborated a constellation of cytokines including interferon gamma and IL-2 which in turn recruited, activated, and sustained second level effector cells (e.g. macrophages and neutrophils) which produced indiscriminant tissue damage including fibrin deposition, thrombus formation, infarction, and bulk ischaemic necrosis. Although the initiation of this $\mathrm{T}$ cell-dependent mechanism possessed exquisite antigen specificity, the execution of this process was antigenically indiscriminant. Although the immune system suceeded in ridding the eye of the lifethreatening neoplasm, a heavy toll was paid in the irreparable damage to juxtaposed normal ocular tissues.

The piecemeal necrotic pattern of tumour rejection exemplified the teleological ideals of immunological effector mechanisms - that is, elimination of a neoplasm without injuring delicate host tissues which are incapable of regeneration. Unlike DTH effector mechanisms, cytolysis by CTL occurred with exquisite antigen specificity during both the induction and execution of the antitumour response. Tumour cells were categorically eliminated while neighbouring normal cells were spared.

Gaining a clear understanding of these immunoregulatory phenomena may permit the implementation of rapidly emerging, novel immunotherapeutic strategies for treating intraocular neoplasms without jeopardising the anatomical integrity of the eye.

The excellent technical assistance of Ms Elizabeth Mayhew and Ms Jessamee Mellon is gratefully appreciated. This work was supported by NIH grants EY05631 and CA30276 and an unrestricted grant from Research to Prevent Blindness, Inc., New York.

Key words: Cytotoxic T Lymphocytes; Delayed Type Hypersensitivity; Ischaemia; Necrosis; T Cell; Tumour. 


\section{References}

${ }^{1}$ Silverstein A: Allergic reactions to the eyè. In: Gell $\mathrm{P}$, Coombs R, Lachmann $\mathrm{P}$ (eds): Clinical Aspects of Immunology. Blackwell Scientific Publishing, Oxford 1975, pp. 1587-98.

${ }^{2}$ Elschnig A: Studien zur sympathischem Ophthalmis: Die antigene Wirkung des Augenpigmentes. Graefes Arch Clin Exp Ophthalmol 1910, 76: 509-46.

${ }^{3}$ Culliton B: Gene therapy: Into the home stretch. Science 1990, 249: 974-6.

${ }^{4}$ Niederkorn J and Meunier P: Spontaneous immune rejection of intraocular tumours in mice. Invest Ophthalmol Vis Sci 1985, 26: 877-84.

${ }^{5}$ Knisely T, Luckenbach M, Fischer B, Niederkorn J: Destructive and nondestructive patterns of immune rejection of syngeneic intraocular tumours. J Immunol 138: 4515-23.

${ }^{6}$ Luckenbach M, Streilein J, Niederkorn J: Histopathologic analysis of intraocular allogeneic tumours in mice. Invest Ophthalmol Vis Sci 1985, 26: $1368-76$.

${ }^{7}$ Niederkorn J and Knisely T: Immunological analysis of a destructive pattern of intraocular tumour resolution. Curr Eye Res 1988, 7: 515-26.

${ }^{8}$ Knisely T and Niederkorn J: Emergence of a dominant cytotoxic $\mathrm{T}$ lymphocyte antitumour effector from tumour-infiltrating cells in the anterior chamber of the eye. Cancer Immunol Immunother 1990, 30: 323-30.

${ }^{9}$ Erickson R, Tachibana D, Herzenberg L, Rosenberg L: A single gene controlling hemolytic complement and a serum antigen in the mouse. J Immunol 1964, 92: 611-5.

${ }^{10}$ Ichikawa T, Kawai M, Uenaka A, Yamamoto H, Gotoh M, Monden M, Mori T, Shiku H, Nakayama E: The role of CD8+ and CD4+ cells in rejection of multiple minor $\mathrm{H}$-disparate skin grafts. Transplantation 1989, 47: 909-10.
${ }^{11}$ Dvorak H, Mihm Jr M, Dvorak A, Barnes B, Manseau E, Galli S: Rejection of first-set skin allografts in man. J Exp Med 1979, 150: 322-37.

${ }^{12}$ Dvorak H, Mihm Jr M, Dvorak A, Johnson R, Manseau E, Morgan E, Colvin R: Morphology of delayed type hypersensitivity reactions in man. I: Quantitative description of the inflammatory response. Lab Invest 1974, 31: 111-30.

${ }^{13}$ Dvorak H, Dvorak A, Manseau E, Wiberg L, Churchill W: Fibrin gel investment associated with line 1 guinea pigs. Role of cellular immunity, myofibroblasts, microvascular damage, and infarction in line 1 tumor regression. JNCI 1979, 62: $1259-66$.

${ }^{14}$ Galli S, Bast Jr R, Bast B, Isomura T, Zbar B, Rapp $H$, Dvorak H: Bystander suppression of tumor growth: Evidence that specific targets and bystanders are damaged by injury to a common microvasculature. J Immunol 1982, 129: 1790-9.

${ }^{15}$ Dvorak H, Dickersin G, Dvorak A, Manseau E, Pyne K: Human breast carcinoma: Fibrin deposits and desmoplasia. Inflammatory cell type and distribution. Microvasculature and infarction. JNCI 1981, 67: 335-45.

${ }^{16}$ Khodadoust A, Roozitalab H, Smith R, Green W: Spontaneous regression of retinoblastoma. Surv Ophthalmol 1977, 21: 467-78.

${ }^{17}$ Sanborn G, Augsburger J, Shields J: Spontaneous regression of bilateral retinoblastoma. $\mathrm{Br} J \mathrm{Oph}$ thalmol 1982, 66: 685-90.

${ }^{18}$ Friedlaender M: Ocular tumors. In: Allergy and Immunology of the Eye. Harper and Row, Hagerstown 1979 , pp. 295-12.

${ }^{19}$ Gangwar D, Jain I, Gupta A, Sharma P: Bilateral spontaneous regression of retinoblastoma with dominant transmission. Ann Ophthalmol 1982, 14: $479-80$.

${ }^{20}$ Char D, Wood I, Huhta K, Rand N, Morita C, Howes Jr E: Retinoblastoma: Tissue culture lines and monoclonal antibody studies. Invest Ophthalmol Vis Sci 1984, 25: 30-40. 